Structural and Magnetic States in Layered Manganites:

An Expanding View of the Phase Diagram

\author{
J.F. Mitchell, J.E. Millburn, C. Ling, D.N. Argyriou, and H.N. Bordallo*
}

Materials Science Division

Argonne National Laboratory, Argonne, IL 60439

The submitted manuscript has been created by the University of Chicago as Operator of Argonne National Laboratory ("Argonne") under Contract No. W-31-109-ENG-38 with the U.S. Department of Energy. The U.S. Government retains for itself, and others acting on its behalf, a paid-up, nonexclusive, irrevocable worldwide license in said article to reproduce, prepare derivative works, distribute copies to the public, and perform publicly and display publicly, by or on behalf of the Government.

Materials Reserach Society Symposium Proceedings, Boston, MA, Nov. 29-Dec. 3, 1999

Work supported by the U.S. Department of Energy, Basic Energy Sciences-Materials Sciences under contract \#W-31-109-ENG-38. 


\section{DISCLAIMER}

This report was prepared as an account of work sponsored by an agency of the United States Government. Neither the United States Government nor any agency thereof, nor any of their employees, make any warranty, express or implied, or assumes any legal liability or responsibility for the accuracy, completeness, or usefulness of any information, apparatus, product, or process disclosed, or represents that its use would not infringe privately owned rights. Reference herein to any specific commercial product, process, or service by trade name, trademark, manufacturer, or otherwise does not necessarily constitute or imply its endorsement, recommendation, or favoring by the United States Government or any agency thereof. The views and opinions of authors expressed herein do not necessarily state or reflect those of the United States Government or any agency thereof. 


\section{DISCLAIMER}

Portions of this document may be illegible in electronic image products. Images are produced from the best available original document. 


\title{
STRUCTURAL AND MAGNETIC STATES IN LAYERED MANGANITES: AN EXPANDING VIEW OF THE PHASE DIAGRAM
}

\author{
J.F. MITCHELL, J.E. MILLBURN, C. LING, D.N. ARGYRIOU AND H. N. BORDALLO* \\ Materials Science Division, Argonne National Laboratory, Argonne, IL 60439 \\ *Intense Pulsed Neutron Source, Argonne National Laboratory, Argonne, II 60439
}

\section{ABSTRACT}

Colossal magnetoresistive (CMR) manganites display a spectacular range of structural, magnetic, and electronic phases as a function of hole concentration, temperature, magnetic field, etc. Although the bulk of research has concentrated on the 3-D perovskite manganites, the ability to study anisotropic magnetic and electronic interactions made available in reduced dimensions has accelerated interest in the layered Ruddlesden-Popper (R-P) phases of the manganite class. The quest for understanding the coupling among lattice, spin, and electronic degrees of freedom (and dimensionality) is driven by the availability of high quality materials. In this talk, we will present recent results on synthesis and magnetic properties of layered manganites from the $\mathrm{La}_{2-2 x} \mathrm{Sr}_{1+2 x} \mathrm{Mn}_{2} \mathrm{O}_{7}$ series in the $\mathrm{Mn}^{4+}$-rich regime $\mathrm{x}>0.5$. This region of the composition diagram is populated by antiferromagnetic structures that evolve from the A-type layered order to G-type "rocksalt" order as $\mathrm{x}$ increases. Between these two regimes is a wide region $(0.7<x<0.9)$ where an incommensurate magnetic structure is observed. The IC structure joins spin canting and phase separation as a mode for mixed-valent manganites to accommodate FM/AF competition. Transport in these materials is dominated by highly insulating behavior, although a region close to $\mathrm{x}=0.5$ exhibits metal-nonmetal transitions and an extreme sensitivity to oxygen content. We suggest two possible explanations for this transport behavior at doping just above $\mathrm{x}=0.5$ : localization by oxygen defects or charge ordering of $\mathrm{Mn}^{3+} / \mathrm{Mn}^{4+}$ sites.

\section{INTRODUCTION}

Colossal magnetoresistive oxides have captured the interest of condensed matter scientists because of their strong coupling among spin, lattice, and charge degrees of freedom. The delicate balance of energy scales among these three aspects of manganite physics gives rise to the dramatic properties observed in the manganites, including insulator-metal (IM) transitions,
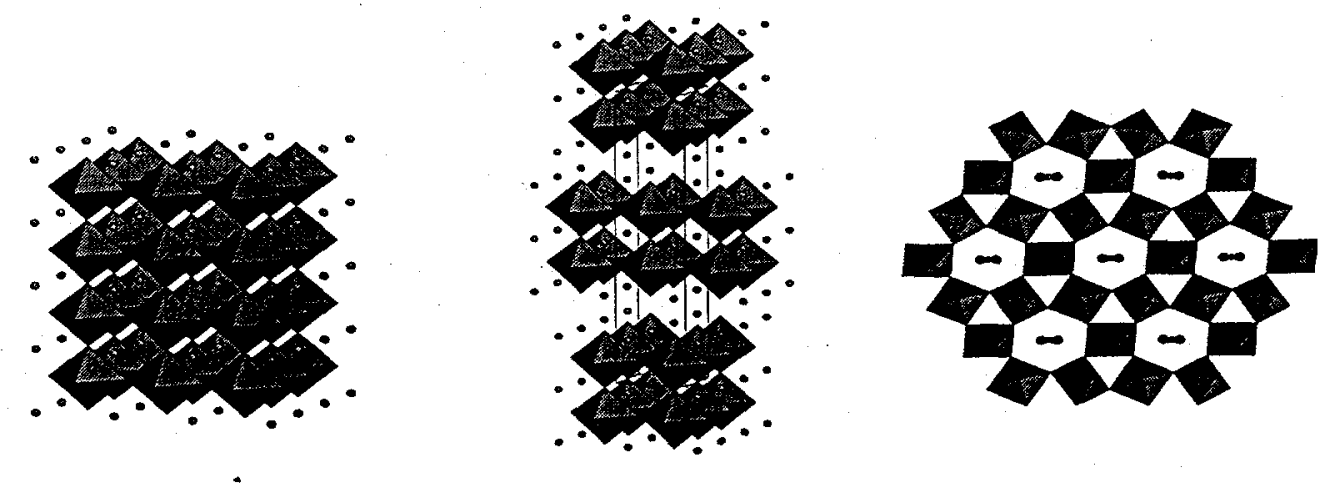

Figure 1. 
competing ferromagnetism (FM) and antiferromagnetism (AF), polaron dynamics, Jahn-Teller distortions, etc.

To date, there are three classes of oxide materials that exhibit colossal magnetoresistance; these three structure types are pictured in Fig. 1. On the left is the well-known perovskite phase, on the right is the pyrochlore structure of $\mathrm{Tl}_{2} \mathrm{Mn}_{2} \mathrm{O}_{7}$, and in the middle is the layered $\mathrm{n}=2$ Ruddlesden-Popper (R-P) phase. All three structure types have corner-linked $\mathrm{MnO}_{6}$ octahedra as their building blocks, although the connectivity of these octahedra is somewhat different in the pyrochlore. It is generally accepted that the mechanism of CMR in the pyrochlore differs from that of the perovskite or layered compounds, as the pyrochlore is not a mixed-valent compound like the other two materials.

Although much of the focus in manganite research has centered around the perovskites, the discovery by Moritomo, et al.[1] of dramatically higher low-field magnetoresistance in the layered manganite $\mathrm{La}_{1.2} \mathrm{Sr}_{1.8} \mathrm{Mn}_{2} \mathrm{O}_{7}$ draws attention to the importance of these reduced dimensionality materials. In addition, the quasi-2D structure is expected to amplify the impact of charge and spin fluctuations in the region $T>T_{C}$. These stronger fluctuations can provide an inroad for probing the critical phenomena associated with the phase transition region, as has been shown recently by Doloc, et al.[2] and Rosenkrantz, et al.[3]

Unfortunately, the range of compositions available in the $\mathrm{R}-\mathrm{P}$ phase $\mathrm{La}_{2 \cdot 2 \mathrm{x}} \mathrm{Sr}_{1+2 x} \mathrm{Mn}_{2} \mathrm{O}_{7}$ has been very limited. Single crystals and powders have only been successfully prepared in singlephase form in the region $0.3<x<0.5,[4-6]$ and materials problems have been noted at each of these endpoints.[7,8] Extending the range of available phase space to $x>0.5$ would provide the opportunity (a) to study the structure of the "baseline" compound $\mathrm{Sr}_{3} \mathrm{Mn}_{2} \mathrm{O}_{7}(\mathrm{x}=1.0)$ which has no Jahn-Teller active $\mathrm{Mn}^{3+}$ sites, and (b) to explore if and where antiferromagnetism and charge ordering impact the physics of the $\mathrm{Mn}^{4+}$ rich region of the phase diagram. We have fully described the crystal and magnetic structure of $\mathrm{Sr}_{3} \mathrm{Mn}_{2} \mathrm{O}_{7}$ in an earlier work. $[9,10]$ In this paper we describe the successful synthesis of the compounds with $\mathrm{x}$ between 0.5 and 1.0 via metastable oxygen deficient intermediates. We then present the structural and magnetic phasa diagram, which shows an evolution of magnetic structure from layered A-type to "rocksalt" G-type via an incommensurate magnetic phase. As is the case in the ferromagnetic materials, lattice and magnetic structures are closely tied. Finally, we comment on the transport characteristics of these materials near $x=0.5$, using the sensitivity to oxygen content to comment on the possible role of defect-induced localization or charge-ordering in this regime.

\section{EXPERIMENTAL}

Polycrystalline samples of $\mathrm{La}_{2-2 x} \mathrm{Sr}_{1+2 x} \mathrm{Mn}_{2} \mathrm{O}_{7}(0.5 \leq \mathrm{x} \leq 1.0)$ were synthesized by a hightemperature, solid state route. $\mathrm{La}_{2} \mathrm{O}_{3}$ (Johnson-Matthey, $99.99 \%$, dried at $1000^{\circ} \mathrm{C}$ immediately before weighing), $\mathrm{SrCO}_{3}$ (Johnson-Matthey, 99.999\%), and $\mathrm{MnO}_{2}$ (Johnson-Matthey, 99.999\%) were combined in stoichiometric ratios and fired in air as powders first at $900{ }^{\circ} \mathrm{C}$ for 24 hours and subsequently at $1050^{\circ} \mathrm{C}$ for an additional 24 hours. Samples were then pressed into $13 \mathrm{~mm}$ disks at $6000 \mathrm{lbs}$ and ramped at $5^{\circ} \mathrm{C} / \mathrm{min}$ to $1650{ }^{\circ} \mathrm{C}$. After 18 hours at synthesis temperature, each sample was quenched by dropping directly into dry ice. As is the case for $\mathrm{Sr}_{3} \mathrm{Mn}_{2} \mathrm{O}_{7-\delta}$ [9], the compounds are metastable and must be rapidly cooled below $1000{ }^{\circ} \mathrm{C}$ to prevent decomposition. As described below, the as-made materials are oxygen deficient To obtain stoichiometric compounds, each sample was annealed for 12 hours at $400^{\circ} \mathrm{C}$ in flowing oxygen. The oxygen content of both as-made and annealed samples was determined both titrimetrically versus standardized sodium thiosulfate solution and thermogravimetrically. Battle, et al. have pointed out the difficulty in preparing single-phase samples of $n=2 R-P$ phases near $x=0.5$. [8] 
We have carefully examined our samples using high resolution synchrotron $x$-ray diffraction and find no evidence of the subtle phase separation these researchers discuss. Apparently the high temperature synthesis allows for more complete reaction and results in single phase samples.

Time-of-flight powder neutron diffraction data were collected on the Special Environment Powder Diffractometer (SEPD) and/or the High Intensity Powder Diffractometer (HIPD) at Argonne National Laboratory's Intense Pulsed Neutron Source (IPNS). The latter instrument is well suited for study of magnetic structures due to its high flux at long d-spacings. Measurements were taken as a function of temperature between $20 \mathrm{~K}$ and $300 \mathrm{~K}$. All crystal structure analysis was performed by the Rietveld method using the GSAS program suite[11]

$\mathrm{X}$-ray synchrotron measurements were made on the bending magnet beamline $12-\mathrm{BM}$ at the Advanced Photon Source (APS). Samples were pulverized and ground to $<35 \mu \mathrm{m}$ particle size. Measurements were taken at an incident energy of $9.2 \mathrm{keV}$ in a $\theta-2 \theta$ mode.

Electrical resistance was measured as a function of temperature on sintered pellets using a standard four-lead technique. Measurements were typically repeated on cooling and heating to identify any thermal hysteresis.

\section{RESULTS AND DISCUSSION}

Synthesis of $\mathrm{La}_{2-2 \mathrm{x}} \mathrm{Sr}_{1+2 \mathrm{x}} \mathrm{Mn}_{2} \mathrm{O}_{7}(0.5<\mathrm{x} \leq 1.0)$ is complicated by the fact that this $\mathrm{n}=2 \mathrm{R}-\mathrm{P}$ compound is not the equilibrium phase in air below $\mathrm{T} \sim 1600^{\circ} \mathrm{C} .[9,12]$. Thus, preparation of these materials requires quenching from synthesis temperatures in excess of $1600{ }^{\circ} \mathrm{C}$ to prevent decomposition into perovskite or $n=1$ R-P phases. At these high temperatures, reduction of $\mathrm{Mn}^{4+}$ to $\mathrm{Mn}^{3+}$ becomes thermodynamically favorable, and the compounds accommodate this reduction by forming oxygen vacancies. Thus, the appropriate formulation for the as-prepared samples is

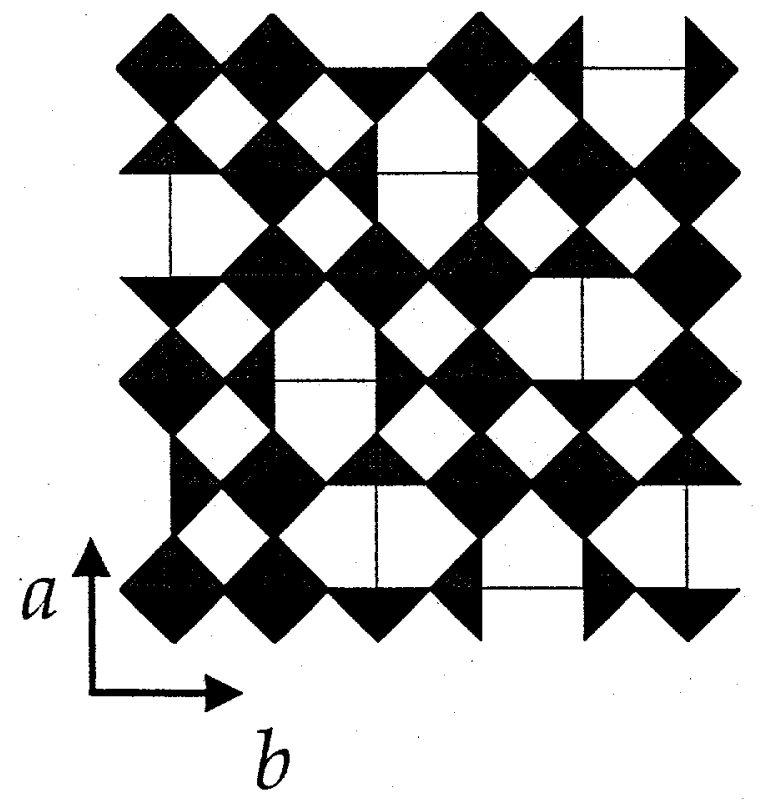
$\mathrm{La}_{2-2 x} \mathrm{Sr}_{1+2 \mathrm{x}} \mathrm{Mn}_{2} \mathrm{O}_{7-\delta}$. As shown in Fig. 2 for the $\mathrm{x}=1.0$ compound, neutron diffraction unambiguously locates these vacancies in the $\mathrm{MnO}_{2}$ conduction planes, forming a mixture of octahedra $\left(\mathrm{Mn}^{4+}\right)$ and square pyramids $\left(\mathrm{Mn}^{3+}\right)$ disordered throughout the structure[9]. It should be noted that the vast majority of $n=2 R$ $P$ phases with oxygen vacancies order these vacancies between the planes, making the manganites described here unusual in their crystal chemistry.[13-14]

Titrimetric analysis of the average $\mathrm{Mn}$ oxidation state versus standardized thiosulfate solution reveals a relationship between the oxygen nonstoichiometry, $\delta$, and the dopant concentration $\mathrm{x}$. This relationship suggests that the synthesis conditions $\left(\mathrm{T}=1650^{\circ} \mathrm{C}, \mathrm{p}\left(\mathrm{O}_{2}\right)=\right.$

Figure 2.

$0.21 \mathrm{~atm}$ ) set the average $\mathrm{Mn}$ oxidation state at 3.5 , i.e. $\left[\mathrm{Mn}^{3+}\right]=\left[\mathrm{Mn}^{4+}\right]=50 \%$. Fig. 3 shows the results of the titrimetic analysis, $\delta$, as a function of $x$ (error in $\delta$ is \pm 0.02 ); TGA on selected compositions agrees within experimental error. Consideration of the chemical formula $\mathrm{La}_{2}$ ${ }_{2 x} \mathrm{Sr}_{1+2 x} \mathrm{Mn}_{2} \mathrm{O}_{7-\delta}$ shows that the average $\mathrm{Mn}$ oxidation state is given by $3+\mathrm{x}+\delta$. Thus, the linear relationship between $x$ and $\delta$ with slope $=1$ implies a constant average Mn oxidation state, and the 
intercept at $x=0.5$ fixes this mean oxidation state at 3.5. It is conceivable that changing the synthesis conditions (e.g., changing $\mathrm{p}\left(\mathrm{O}_{2}\right)$ ) could shift this mean oxidation state.

Also shown on Fig. 3 are the results of titration on oxygen annealed samples. The outcome of this oxygen treatment has been to fill all of the oxygen vacancies, generating a stoichiometric $n=2$ R-P phase in the previously inaccessible region of composition space. It is important to note that these new compositions are metastable. Indeed, heating these stoichiometric compounds to $1400{ }^{\circ} \mathrm{C}$ in air slowly decomposes them into phase mixtures of $\mathrm{n}=1,2 \mathrm{R}-\mathrm{P}$ phases and perovskite.

\section{$\underline{\text { Magnetism }}$}

Rietveld refinement of neutron powder diffraction data measured on the stoichiometric compounds $(\delta=0)$ reveals an evolution of the magnetic structure from layered A-type to "rocksalt" G-type as $x$ increases from 0.5 toward 1.0. However, this evolution is not smooth, as shown by the magnetic phase diagram of the $\mathrm{La}_{2}$ ${ }_{2 x} \mathrm{Sr}_{1+2 x} \mathrm{Mn}_{2} \mathrm{O}_{7.0}$ system in Fig. 4. In this diagram, the Néel temperatures are determined by the onset of intensity in AF peaks. Focusing only on the region $\mathrm{x}>0.5$, the diagram shows four regions: A-type, no long-range order, incommensurate (IC), and G-type. This IC structure is derived as a modulation of the $G$ type structure and represents a new response to the FM/AF competition in manganites.

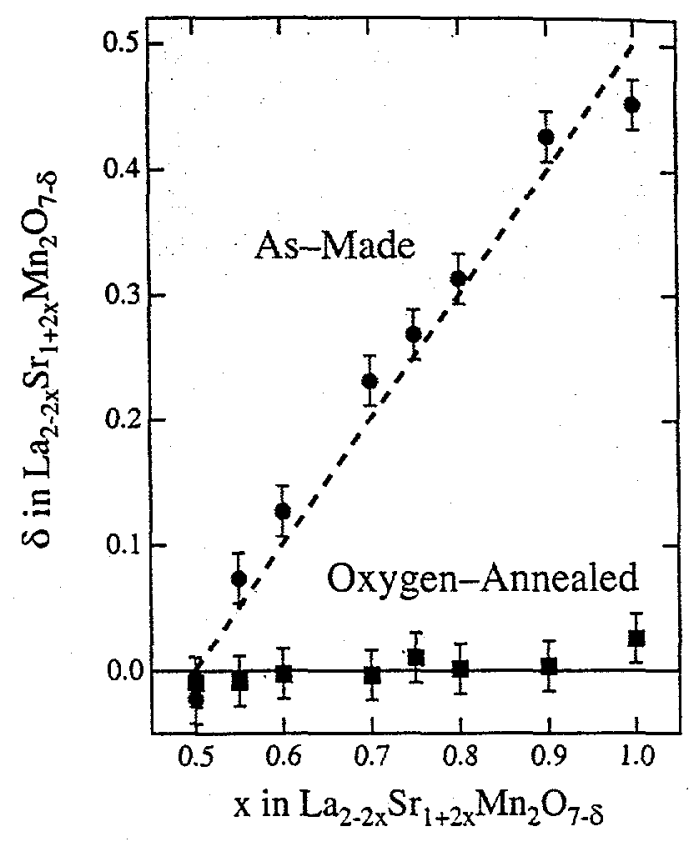

Figure 3.

Fig. 5 shows both the room temperature and $8 \mathrm{~K}$ neutron powder diffraction patterns of $\mathrm{Sr}_{3} \mathrm{Mn}_{2} \mathrm{O}_{7.0}$, the $\mathrm{x}=1.0$ endpoint composition. The presence of extra reflections below the Néel

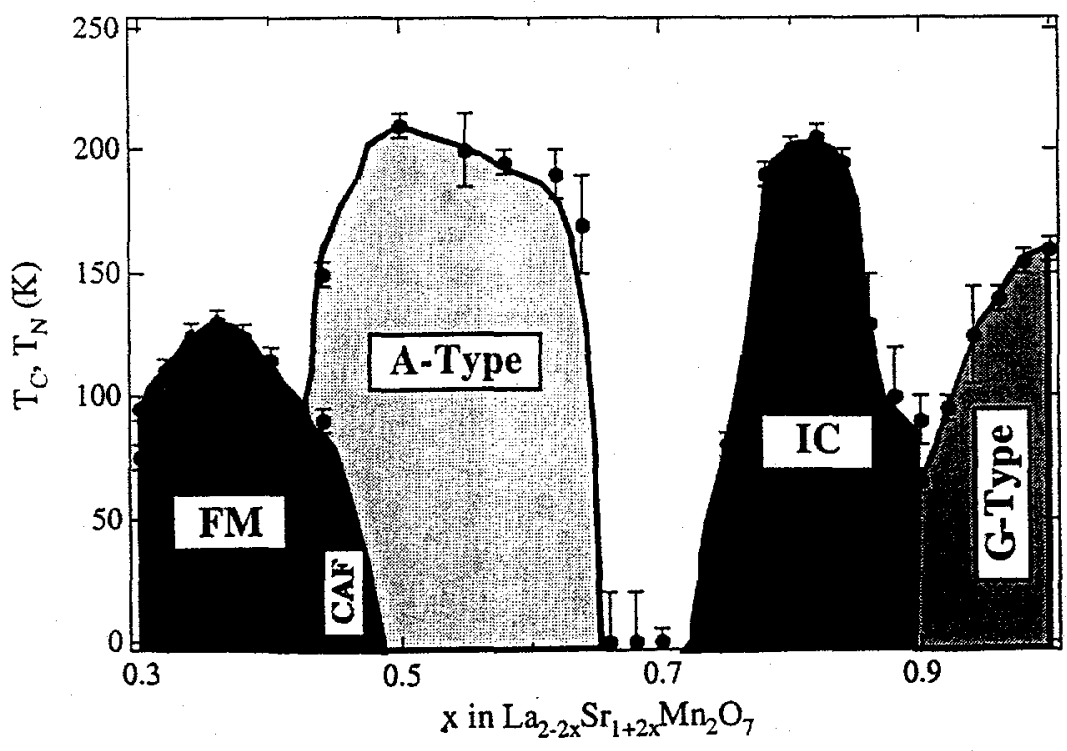

Figure 4. temperature of $160 \mathrm{~K}$ is consistent with an $\mathrm{AF}$ supercell $a_{\mathrm{m}}=2 a_{\mathrm{c}}, c_{\mathrm{m}}=c_{\mathrm{c}}$. $\left(a_{\mathrm{c}}\right.$ and $c_{\mathrm{c}}$ are the $I 4 / \mathrm{mmm}$ crystallographic unit cell constants). The $\mathrm{AF}$ ordering temperature is approximately $100 \mathrm{~K}$ lower than that of the cubic perovskite $\mathrm{SrMnO}_{3},[15]$ reflecting the quasi-2D nature of the layered compound. The refined magnetic structure in Fig. 5 reveals all AF $\mathrm{Mn}-\mathrm{Mn}$ neighbor interactions-a "rocksalt" structure"-and a c-axis orientation of the moment. The magnetic 
structure results from $t_{2 g}{ }^{3}-\mathrm{O}_{2 p}-t_{2 g}{ }^{3}$ superexchange in this undoped parent compound.[19] However, in tetragonal symmetry this magnetic structure is frustrated. The spin labeled with an arrow in one bilayer sees in the neighboring bilayer a square of Mn ions with two "up" spins and two "down" spins across the diagonal of this square. This degeneracy suggests that the true symmetry of the magnetic phase may be lower (e.g., orthorhombic), but the resolution of the neutron data is inadequate to reveal any such symmetry-breaking at this composition.

However, consideration of the entire series from $x=0.5$ to $x=1.0$ clarifies the connection

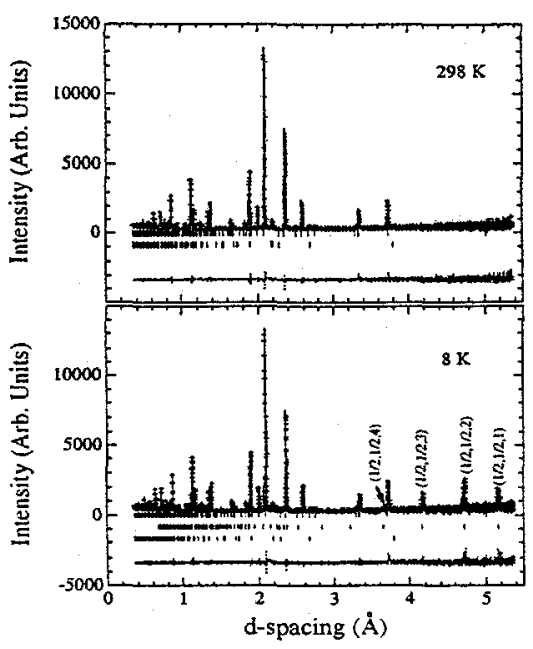

Figure 5.

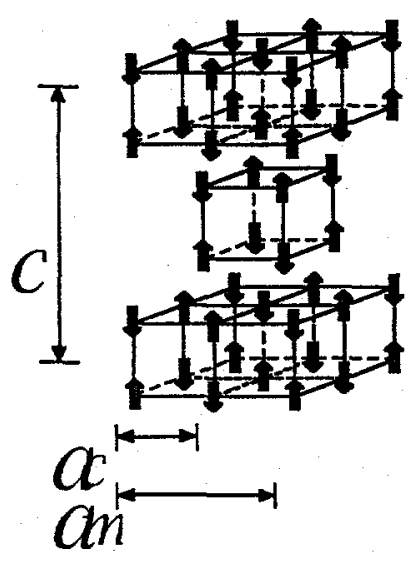
between crystal and magnetic structures by revealing the anticipated symmetry lowering. Fig. 6 shows the variation of the lattice constants with $\mathrm{Sr}$ content as determined by neutron powder diffraction at $300 \mathrm{~K}$ (circles) and at $20 \mathrm{~K}$ (triangles). At room temperature, all of the samples are tetragonal, but at low temperature samples with compositions between $\mathrm{x}=0.74$ and 0.92 become orthorhombic. Previous to this

work, $\mathrm{n}=2$ R-P phases in the $\mathrm{La}_{2-2 \mathrm{x}} \mathrm{Sr}_{1+2 \mathrm{x}} \mathrm{Mn}_{2} \mathrm{O}_{7}$ series have only been found in tetragonal symmetry. As will be discussed shortly, this orthorhombic distortion is found in the same composition space where magnetic order based on the G-type magnetic structure is found (either true G-type or an incommensurate structure based on G-type). Furthermore, a highresolution $\mathrm{x}$-ray measurement at $300 \mathrm{~K}$ for an $\mathrm{x}=0.94$ sample (previously cooled to $20 \mathrm{~K}$ ) shows coexistence between orthorhombic and tetragonal phases. Additional temperature dependent, high resolution measurements will be required to properly map out the relationship between the structural and magnetic phase lines, in particular near $x=1.0$. Nonetheless, the current results support the hypothesis that the Gtype magnetic structure is connected to the orthorhombic phase. At the other end of the region, near $x=0.5$, the A-type antiferromagnetic structure is stable. As pointed out by various researchers, $[6,16,17]$ this structure consists of ferromagnetically coupled $\mathrm{MnO}_{2}$ sheets coupled antiferromagnetically within a given bilayer with

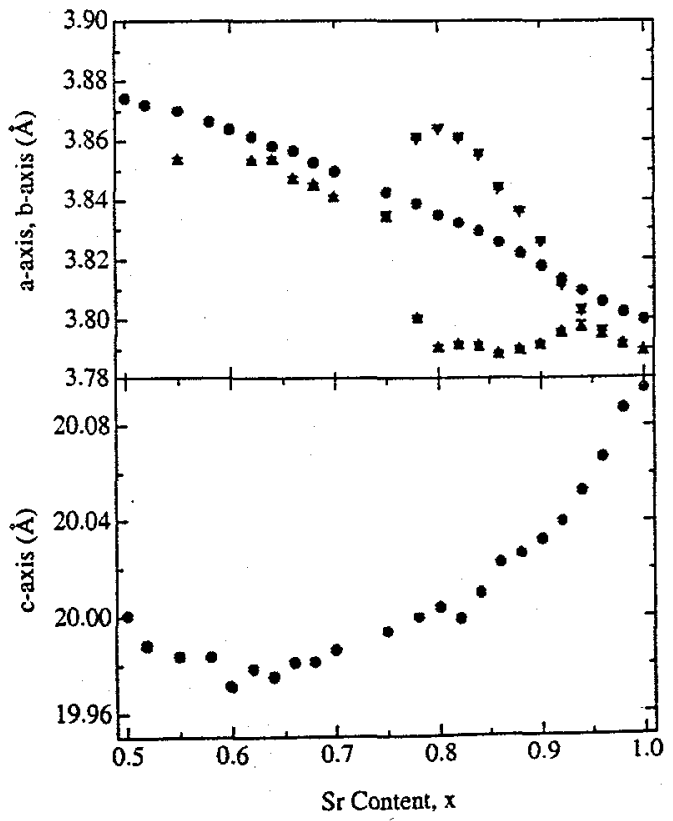

Figure 6. 


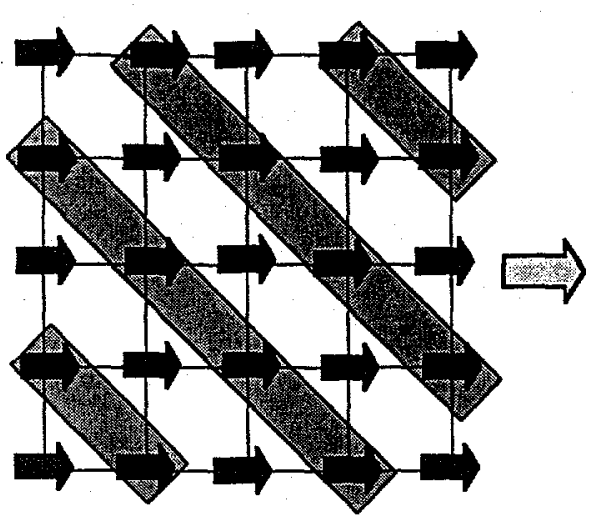

A-type $(\varepsilon=0.0)$

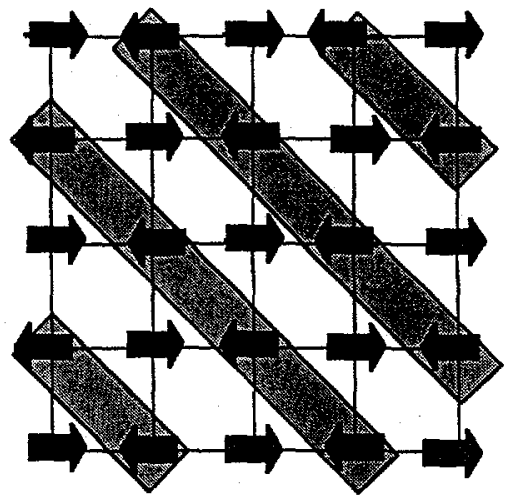

G-type $(\varepsilon=0.5)$

Figure 7.

the Mn spins lying in the $a-b$ plane. This magnetic structure is consistent with an ordering of the $x^{2}-y^{2}$ orbitals on the $\mathrm{Mn}$ atoms in the $a-b$ plane. [18] Our investigations have shown that this structure type is stable up to $\mathrm{x} \sim 0.65$. In this region $\mathrm{T}_{\mathrm{N}}$ is remarkably insensitive to $\mathrm{Sr}$ concentration, varying from $200 \mathrm{~K}$ at $\mathrm{x}=0.5$ to $175 \mathrm{~K}$ at $\mathrm{x}=0.64$ before plummeting dramatically to $<20 \mathrm{~K}$ at $\mathrm{x}=0.66$.

A gap exists between $x=0.66$ and $x=0.72$ where no $A F$ reflections are observed above 20 $\mathrm{K}$, the lowest temperature measured by neutrons $(5 \mathrm{~K}$ in the case of $\mathrm{x}=0.70)$. This gap is unprecedented in the manganite field and probably reflects the competing AF and FM interactions at the borders of the A- and G-type structures. At this time we have no detailed microscopic explanation for why such a gap appears. However, there are structural features that may shed light on this question. As shown in Fig. 6, the $c$-axis exhibits a shallow minimum between $x=0.6$ and 0.7. In addition, the $\mathrm{Mn}-\mathrm{O}$ bond lengths (not shown) also have a cross-over in this region, with the $\mathrm{MnO}_{6}$ octahedra becoming completely regular at $\mathrm{x}=0.62-0.64$. Such structural effects are potential signatures of orbital ordering crossovers, which may be important for understanding the magnetic structure of these compositions. Clearly additional experimental and theoretical work are required to understand this particular region of composition space.

Finally, we consider the IC magnetic structure found between $x=0.72$ and $x=0.90$ and the relationship this structure bears to the A-type and G-type magnetic structures. In this region, Rietveld refinement shows that the moment lies in the $a-b$ plane (the crossover is at $x \sim 0.94$ ). Fig. 7 shows schematically how the A- and G-type structures are related in a given $\mathrm{MnO}_{2}$ plane; in both structures this plane will be AF coupled to the other member of the bilayer. Simply flipping the direction of the spins in alternate (110) planes changes the A-type to the G-type structure. Thus, the G-type structure may be considered as a $q=(1 / 2,1 / 2,0)$ modulation of the Atype structure. This can be made more quantitative by introducing an incommensurability parameter, $\varepsilon$, which takes the value $\varepsilon=0$ for the A-type structure and $\varepsilon=0.5$ for the G-type structure. An intermediate value for $\varepsilon$ gives rise to the IC structure. Fig. 8 shows a comparison of the selected portions of the neutron powder diffraction pattern for $x=0.55$ (A-type) and $x=0.92$ (G-type) samples below $T_{N}$ with several magnetic peaks indexed. These patterns are clearly distinct, yet both can be related through the construction described above. Furthermore, note the three doubly degenerate peaks (degenerate because $-\varepsilon=\varepsilon=1-\varepsilon$ for $\varepsilon=1 / 2$ ). As shown in Fig. 8 , these peaks split as $\varepsilon$ departs from 0.5 in the IC phase; here for $\mathrm{x}=0.82, \varepsilon \sim 0.4$.

Fig. 9 shows a preliminary model of the IC structure as a commensurate approximation with $\varepsilon=0.4$. The key features of the model are the spin rotation angle that connects the $A-$ and $G$ type structures and the pairwise interactions that are predominantly AF. With $\varepsilon=0.4$, the repeat 
unit is $5 a_{\mathrm{c}}$. Starting at any site, moving to the neighbor on the right or above is accompanied by a rotation of $144^{\circ}$; five such moves returns the spin to its original configuration. If this angle were $0^{\circ}$, the A-type structure would be produced; if it were $180^{\circ}$, the G-type structure would result. Presumably, any arbitrary value of this rotation angle should be allowed. However, in all cases we examined $(0.75<\mathrm{x}<$ 0.9 ), a value of $\varepsilon$ close to 0.4 is observed, despite $\mathrm{T}_{\mathrm{N}}$ varying from $80 \mathrm{~K}$ at $\mathrm{x}=0.75$ to 205 $\mathrm{K}$ at $\mathrm{x}=0.82$. With $\varepsilon \sim 0.4$, the magnetic structure should closely approximate G-type $(\varepsilon=0.5$ ), and indeed the pairwise interactions between $\mathrm{Mn}$ spins are all close to AF as expected in the G-type phase. The weak FM component presumably arises because of the presence of doped electrons generating $\mathrm{Mn}^{3+}-\mathrm{O}-\mathrm{Mn}^{4+} \mathrm{FM}$ double-exchange interactions.[19] This structure is quite unlike the canted state predicted by DeGennes[20] or a phase separation, both of which have $M \neq 0$. In contrast, compounds in this region adopt the complicated antiferromagnetic $(M=0)$ IC structure to accommodate the competition between AF and FM exchange.

\section{Transport}

In the 3-D perovskite $\mathrm{La}_{1-\mathrm{x}} \mathrm{Ca}_{\mathrm{x}} \mathrm{MnO}_{3}$ (LCMO), the region $\mathrm{x}>0.5$ is characterized by insulating compounds undergoing charge order transitions whose existence displays little

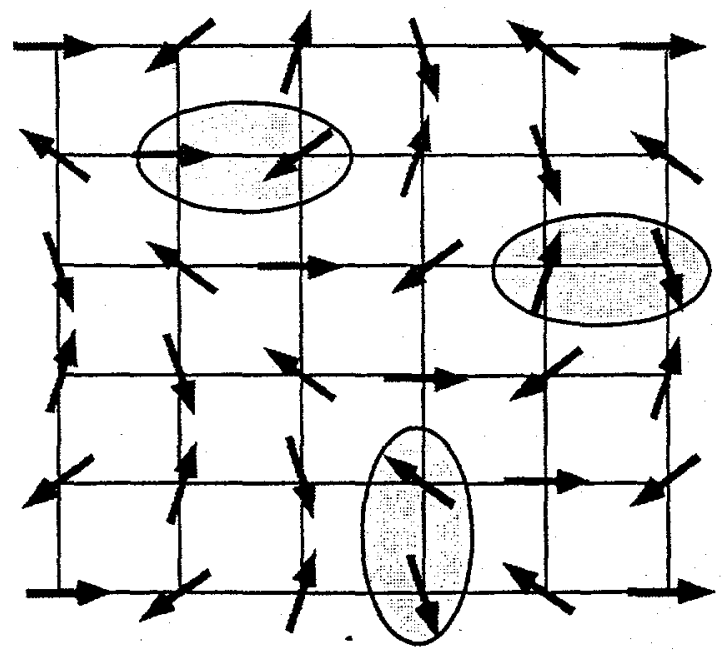

Figure 9.
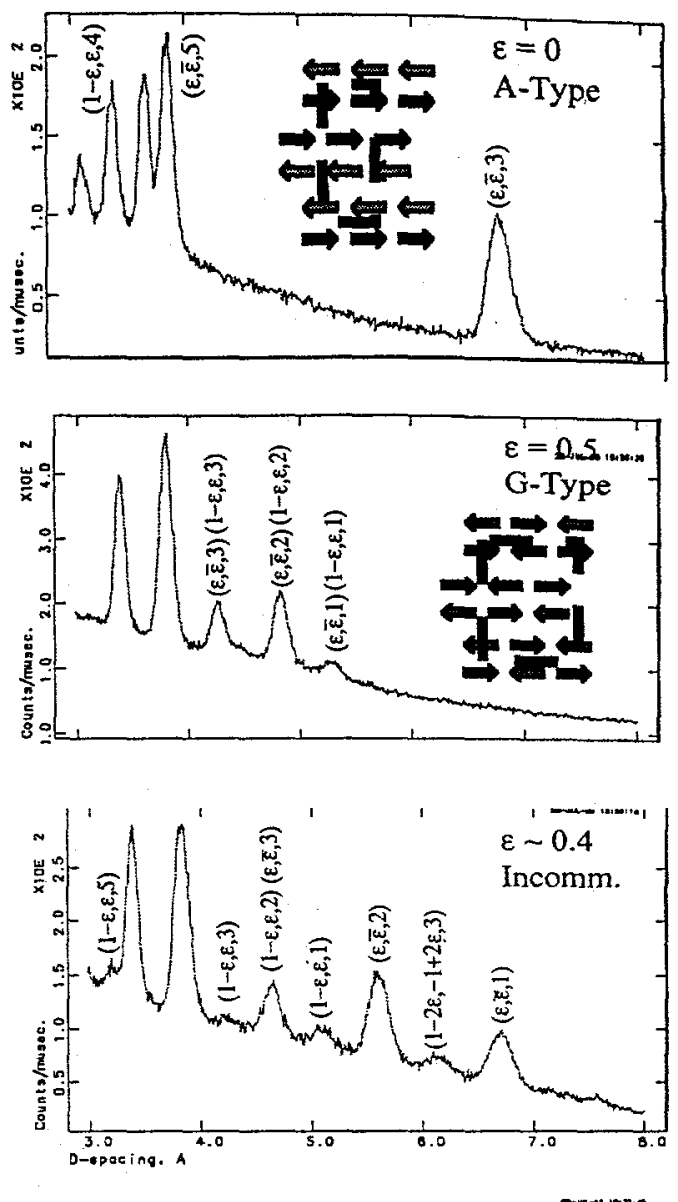

Figure 8 . sensitivity to a $\mathrm{Mn}^{3+} / \mathrm{Mn}^{4+}$ ratio differing from unity.[21] On the other hand, $\mathrm{La}_{1-x} \mathrm{Sr}_{x} \mathrm{MnO}_{3}$ (LSMO) is metallic out to its solubility limit at $\mathrm{x} \sim 0.6$. The reduced dimensionality in the $\mathrm{La}_{2}$ ${ }_{2 x} \mathrm{Sr}_{1+2 x} \mathrm{Mn}_{2} \mathrm{O}_{7}$ system results in transport behavior unlike either of these cases. The majority of the compounds are highly insulating but do not show no the signature of charge-ordering seen in LCMO. On the other hand, a narrow composition region near $x=0.5$ shows metal-nonmetal transitions and an extreme sensitivity to oxygen content. Using electron diffraction, other researchers have shown the presence of charge-ordering in the $x=0.5$ compound $\mathrm{LaSr}_{2} \mathrm{Mn}_{2} \mathrm{O}_{7}$. [22] Based on 
transport measurements, we suggest that similar behavior exists out to $x \sim 0.6$, but that unlike the case of LCMO this property may be highly sensitive to the $\mathrm{Mn}^{3+} \mathrm{Mn}^{4+}$ ratio.

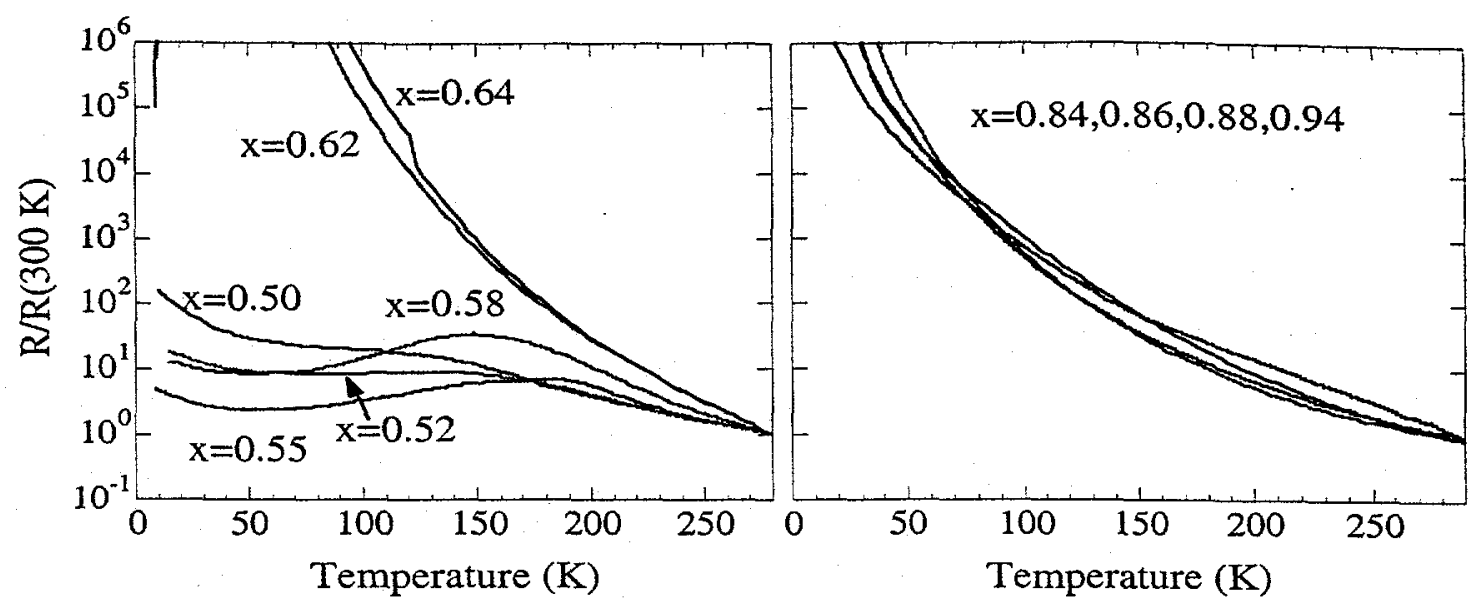

Figure 10.

The transport characteristics of $\mathrm{La}_{2-2 x} \mathrm{Sr}_{1+2 \mathrm{x}} \mathrm{Mn}_{2} \mathrm{O}_{7}(\mathrm{x}>0.5)$ are divide cleanly into two classes: those that show metal-nonmetal transitions, and those that are extremely insulating. Furthermore, the insulating compounds do not show the typical signature of charge ordering seen in perovskites: an abrupt change in $d R / d T$.[23] Fig. 10 shows resistance measurements on several of the stoichiometric $(\delta=0.0)$ compounds. As shown in left panel, samples in the range $0.5 \leq x<$ 0.6 are far less resistive than their counterparts at $x>0.6$. Furthermore, these compounds near $\mathrm{x}=0.5$ typically show a maximum in resistance between $150-200 \mathrm{~K}$, similar to the behavior seen by others in the $x=0.5$ compound.[22]. Samples with higher doping levels (shown in the right panel) are all highly resistive. As shown by other investigators, charge-ordering in perovskites (e.g., LCMO, $\mathrm{Nd}_{1-\mathrm{x}} \mathrm{Sr}_{x} \mathrm{MnO}_{3}, \mathrm{Pr}_{1-\mathrm{x}} \mathrm{Ca}_{\mathrm{x}} \mathrm{MnO}_{3}$ ) causes a sudden change in the slope of $R(T)$ at the charge-ordering temperature. In the case of the $\mathrm{La}_{2-2 \mathrm{x}} \mathrm{Sr}_{1+2 \mathrm{x}} \mathrm{Mn}_{2} \mathrm{O}_{7}$ compounds, $R(T)$ is smoothly varying in the entire temperature region measured,[24] suggesting either that charge ordering is not playing a significant role in the physics of these layered compounds or that its signature is obscured in the transport measurement. In the former case, the localization presumably results from strong AF exchange producing a Mott-Hubbard insulator. Diffraction measurements showing the presence or absence of superlattice reflections will be required to distinguish between these two possibilities.

Charge-ordering of equal numbers of $\mathrm{Mn}^{3+}$ and $\mathrm{Mn}^{4+}$ in the $\mathrm{LaSr}_{2} \mathrm{Mn}_{2} \mathrm{O}_{7}$ system is quite unlike that found in perovskites, as it is only stable in a narrow temperature window between 100 $\mathrm{K}$ and $200 \mathrm{~K}$. It is currently believed that the A-type AF structure competes with charge ordering (which prefers a CE-type magnetic structure) and eventually wins out at low temperature.[25]. The resistive maximum seen in the samples $0.5<\mathrm{x}<0.6$ resembles the $\mathrm{x}=0.5$ data and may imply that a similar effect is at play in this region as well. By exploiting the oxygen nonstoichiometry available in our materials we can comment on the impact of oxygen vacancies on transport and conjecture on the doping range where charge ordering may impact transport properties.

Fig. 11 shows $R(T)$ for two samples with $\mathrm{x}=0.55$ : as-made with $\delta=0.05(2)$ and annealed with $\delta=0.00(2)$. (A second annealed sample is also plotted to demonstrate reproducibility.) The dramatic difference between these curves demonstrates the high sensitivity of transport to 
oxygen content. In this case, an oxygen vacancy concentration of $\sim 1 \%$ precipitates this change, which may arise because the vacancies are located in the $\mathrm{MnO}_{2}$ conduction planes.

As shown in Fig. 3 , the as-made samples contain oxygen vacancies whose concentration varies linearly with the Sr content. The structural implication of this variation is that the ratio of $\mathrm{Mn}^{4+} \mathrm{O}_{6}$ octahed-

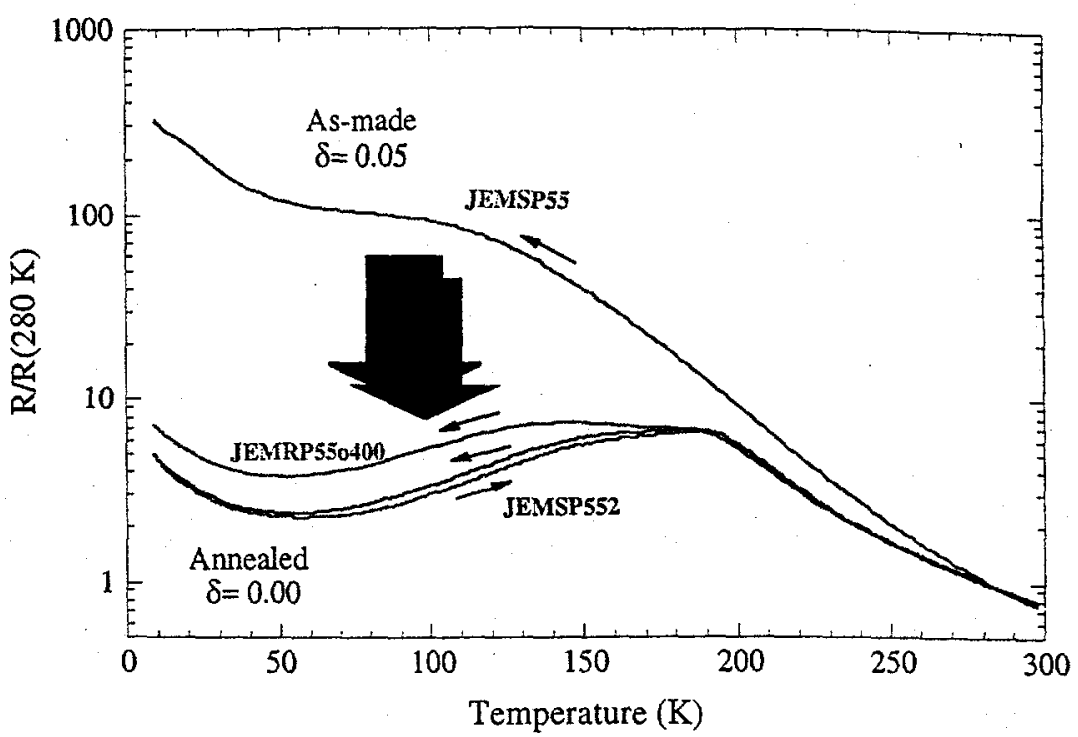

Figure 11.

ra to $\mathrm{Mn}^{3+} \mathrm{O}_{5}$ square pyramids varies systematically with $\mathrm{x}$, as well. This variation is shown graphically in Fig. 12. The solid lines labeled "octa" and "pyr" are based on a model in which

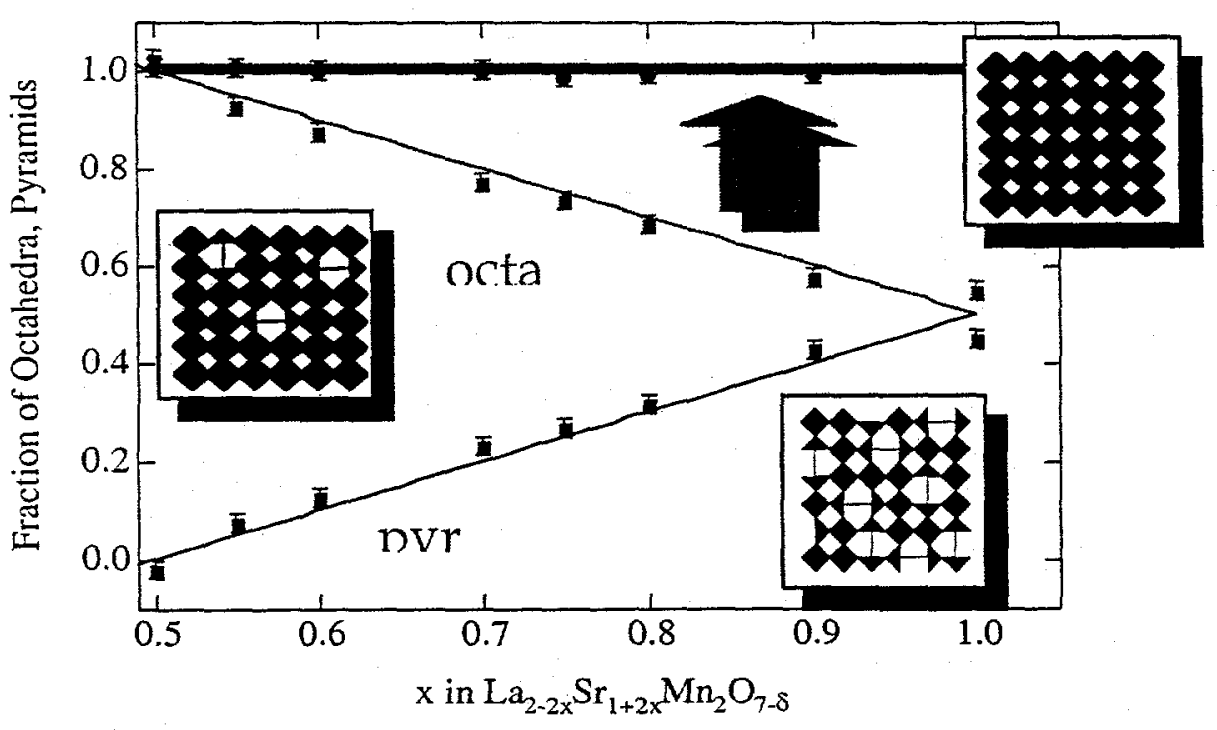
each vacancy removed from the $\mathrm{MnO}_{2}$ planes converts two octahedra into two square pyramids while keeping the mean $\mathrm{Mn}$ oxidation state fixed at 3.5. The points on these lines are based on the measured oxygen content of the compounds in the series and fit well to the model. Naturally, a direct determination of the fraction of octahed-

Figure 12.

ra and pyramids would be desirable. Unfortunately the oxygen vacancies are disordered, precluding an actual measurement of these quantities by diffraction techniques. It is conceivable that the defects act as randomly distributed scattering sites, which in the reduced dimension of the layered compounds are likely to precipitate localization. For $x \sim 0.5$ there are few such defects so resistance intermediate between $x=0.50$ and $x>0.60$ results.

An alternative possibility for explaining the transport behavior rests on the conjecture tha charge ordering in layered manganites is only possible when $\mathrm{Mn}^{3+} \mathrm{Mn}^{4+}=1$. The as-made $x=0.55$ compound contains 0.05 oxygen vacancies per formula unit. Recalling Fig. 3 above, this composition implies a mean $\mathrm{Mn}$ oxidation state of 3.5 , or $\mathrm{Mn}^{3+} / \mathrm{Mn}^{4+}=1$. Such a ratio is also 
found in the $\mathrm{x}=0.50$ sample, which has no oxygen vacancies. As shown in Fig. 13, the $R(T)$ curves for the $x=0.5(\delta=0.0)$ sample and the $x=0.55(\delta=0.05)$ sample are qualitatively similar, yet both differ significantly from the $\mathrm{x}=0.55(\delta=0.0)$ sample, which has a clearly defined maximum

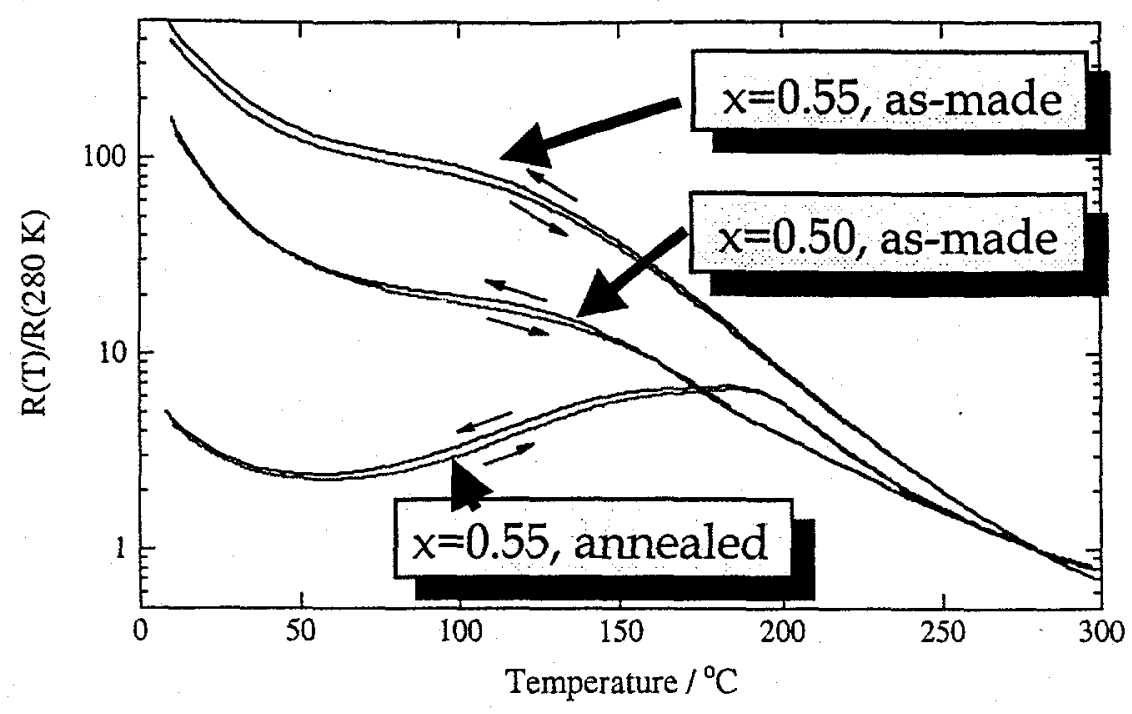

in $R$ near $200 \mathrm{~K}$.' Thus, a picture develops in which charge-ordering, which has been established in the $\mathrm{x}=0.5$ composition, is also stable in the oxygen deficient compounds $\mathrm{x}<$ 0.6. The charge ordering then disappears when the oxygen vacancies are filled. The vacancy-free materials with excess carriers $\left(\mathrm{Mn}^{3+} / \mathrm{Mn}^{4+}<1\right)$

Figure 13.

then exhibit a pronounced nonmetal-metal transition in the absence of the charge-ordering.

There are several constraints implicit in this model beyond the requirement that $\mathrm{Mn}^{3+} / \mathrm{Mn}^{4+}=1$ for charge ordering to be stable. First, it requires that charge ordering be robust to a small oxygen vacancy concentration $(\delta<0.1)$. Second, it implies that small carrier concentrations beyond $\mathrm{x}=0.50$ result in "metallic" like behavior, a feature not seen in LCMO perovskites. Finally, it requires that more highly doped compounds remain insulators even when oxygen stoichiometric, presumably because of a competing AF Mott-Hubbard ground state found at higher $\mathrm{Mn}^{4+}$ concentrations. Despite the speculative nature of this model, the experiments required to test it are clear. X-ray or electron diffraction patterns of $x=0.5-0.6$ asmade materials should all show charge-ordering superlattice reflections in the temperature range $100-200 \mathrm{~K}$, as should the oxygen-annealed sample of $x=0.5(\delta=0.0$ for this composition both as-made or annealed). However, the patterns of the oxygen-annealed samples with $x>0.5$ should show no such superlattice reflections.

\section{SUMMARY}

We have successfully synthesized $n=2 \mathrm{R}-\mathrm{P}$ phases in the $\mathrm{La}_{2-2 \mathrm{x}} \mathrm{Sr}_{1+2 \mathrm{x}} \mathrm{Mn}_{2} \mathrm{O}_{7}$ series for $\mathrm{x}>$ 0.5 using a two-step process whose intermediate state is an oxygen deficient material. Efforts on understanding the structural, magnetic, and electronic behavior of the series are in their early stages. Nonetheless, despite the absence of ferromagnetic ground states, it is clear that the variety of magnetic and electronic phases in this $\mathrm{Mn}^{4+}$-rich region compares favorably to that of the previously known composition space between $x=0.3$ and $x=0.5$.

As in the perovskites and the known layered manganites, structure and magnetism are tightly coupled. In particular, we have determined that the magnetic structure evolves from the A-type layered antiferromagnetic structure through an unprecedented "gap" in which no magnetism is observed, to an incommensurate phase, and finally to a G-type "rocksalt" antiferromagnet near the $x=1.0$ endpoint. The IC structure joins spin canting and phase 
separation as a mode for mixed-valent manganites to accommodate FM/AF competition. The existence of a tetragonal-orthorhombic phase line for $x>0.72$ (i.e., in the IC and G-type regions) provides the mechanism necessary to relieve a magnetic degeneracy inherent to the G-type structure in the tetragonal symmetry of the paramagnetic phase. Likewise, the appearance of regular $\mathrm{MnO}_{6}$ octahedra in the magnetic "gap" region may signify an orbital ordering crossover.

Transport measurements show that the majority of compositions in the $\mathrm{Mn}^{4+}$-rich region are highly insulating $(0.6<x<1.0)$. However, for $x \sim 0.5$ transport is characterized by metalnonmetal transitions and an extreme sensitivity to small $O$ vacancy concentrations. Our preliminary results are consistent with a model in which charge-ordering is stable only at $\mathrm{Mn}^{3+} \mathrm{Mn}^{4+}=1$, but further measurements will be required to verify this thesis.

\section{ACKNOWLEDGMENTS}

The authors thank Mark Beno, Guy Jennings, and Jennifer Linton for assistance with the synchrotron measurements, and Simine Short for assistance at SEPD. This work was supported the the U.S. Department of Energy, Basic Energy Sciences-Materials Sciences under Contract W-31-109-ENG-38 and W-7405-ENG-36 (DNA)

\section{REFERENCES}

1. Y. Moritomo, A. Asamitsu, H. Kuwahara and Y. Tokura Nature 380, 141 (1996).

2. L. Vasiliu-Doloc, S. Rosenkranz, R. Osborn, S. K. Sinha, J. W. Lynn, J. Mesot, O. H. Seeck, G. Preosti, A. J. Fedro and J. F. Mitchell Phys. Rev. Lett. 83, 4393 (1999).

3 S. Rosenkranz, R. Osborn, J.F. Mitchell, L. Vasiliu-Doloc, J. Lynn, S.K. Sinha, D.N. Argyriou J. Appl. Phys. 83, 7348 (1998).

4. R. Seshadri, c. Martin, M. Hervieu, B. Raveau, and C.N.R. Rao Chem. Mater. 73, 1097 (1997).

5. M. Medarde, J. F. Mitchell, J. E. Millburn, S. Short and J. D. Jorgensen Phys. Rev. Lett. 83, 1223 (1999).

6. K. Hirota, Y. Moritomo, H. Fujioka, M. Kubota, H. Yoshizawa and Y. Endoh J. Phys. Soc. Jpn. 67, 3380 (1998).

7. D.N. Argyriou, J.F. Mitchell, P.G. Radaelli, H. N. Bordallo, D.E. Cox, M. Medarde and J.D. Jorgensen Phys. Rev. B 59, 8695 (1999).

8. P.D. Battle, D.E. Cox, M.A. Green, J.E. Millburn, L.E. Spring, P.G. Radaelli, M.J. Rosseinsky and J.F. Vente Chem. Mater. 9, 1042 (1997).

9. J.F. Mitchell, J.E. Millburn, M. Medarde, S. Short, J.D. Jorgensen and M.T. FernándezDiaz J. Solid State Chem. 141, 599 (1998).

10. J.F. Mitchell, J.E. Millburn, M. Medarde, D.N. Argyriou and J.D. Jorgensen J. Appl. Phys. 85, 4352 (1999).

11. A.C. Larson and R.B. Von Dreele, General Structural Analysis System, Los Alamos Internal Report No. 86-748, 1990.

12. N. Mitzutani, A. Kitazawa, O. Nobuyuki and M. Kato J. Chem. Soc. (Jpn.) Ind. Ed. 73, 1097 (1970).

13. M. Itoh, M. Shikano, H. Kawaji and T. Nakamura Solid State Comm. 80, 545 (1991).

14. S.E. Dann, M.T. Weller and D.B. Currie J. Solid State Chem. 97, 179 (1992).

15. T. Takeda and S. Ohara J. Phys. Soc. Jpn. 37, 275 (1974).

16. M. Kubota, H. Yoshizawa, Y. Moritomo, H. Fujioka, K. Hirota, Y. Endoh J. Phys. Soc. Jpn 68, 2202 (1999). 
17. M. Kubota, H. Fujioka, K. Ohoyama, K. Hirota, Y. Moritomo, H. Yoshizawa and Y. Endoh J. Phys. Chem. Solids 60, 11261 (1999).

18. R. Maezono and N. Nagaosa cond-matt/9904427 (1999).

19. J.B. Goodenough, Magnetism and the Chemical Bond (Interscience Publishers, New York, 1963).

20. P.G. DeGennes, Phys. Rev. 118, 141 (1960)

21. A.P. Ramirez, P. Schiffer, S-W. Cheong, C.H. Chen, W. Bao, T.T.M. Palstra, P. L. Gammel, D.J. Bishop and B. Zegarski Phys. Rev. Lett. 76, 3188 (1996).

22. J.Q. Li, Y. Matsui, T. Kimura and Y. Tokura Phys. Rev. B 57, R3205 (1998).

23. M. Roy, J.F. Mitchell, A. Ramirez, P. Schiffer J. Phys.: Cond. Matt. 11, 4843 (1999).

24. The discontinuity in the $\mathrm{x}=0.64$ curve is an experimental artifact.

25. D.N. Argyriou, private communication. 